\title{
Niños migrantes, ¿una permanente violación a sus derechos?
}

\section{Migrant Children, a Permanent Violation of your Rights?}

Rolando Castillo Santiago * https://orcid.org/0000-0001-8764-9168

http://dx.doi.org/10.21503/lex.v18i25.2096

*Profesor investigador de la Universidad Juárez Autónoma de Tabasco. Abogado, maestro en Derecho y doctor en Estudios Jurídicos, miembro del PNCP-CONACYT, México.

Correo electrónico: myc_abogado@me.com

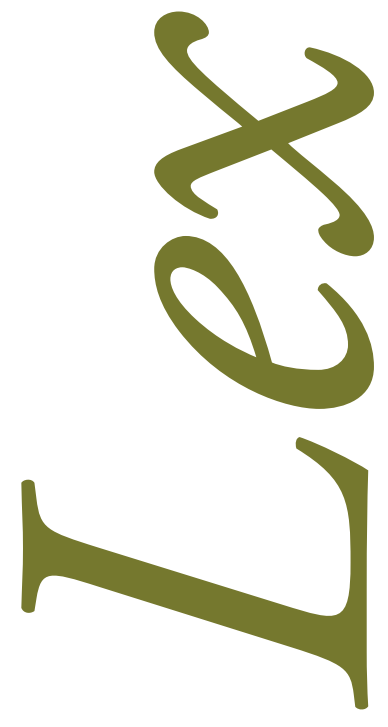

(c) (C) Los autores. Artículo publicado por la Revista Lex de la Facultad de Derecho y Ciencias Políticas de la Universidad Alas Peruanas. Este es un artículo de acceso abierto, distribuido bajo los términos de la Licencia Creative Commons Atribución-No 


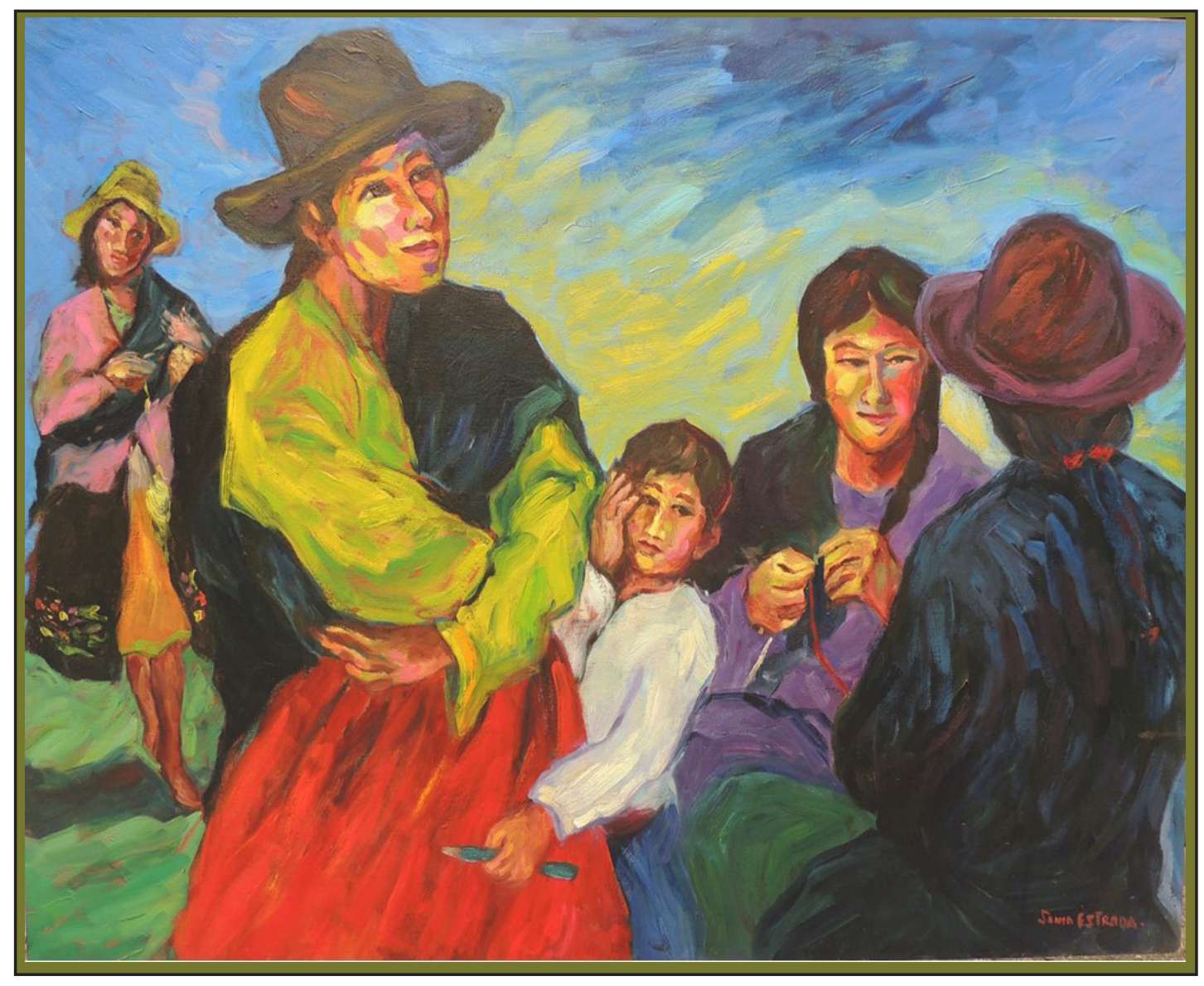

Días y flores. Óleo sobre lienzo 81 x $166 \mathrm{~cm}$. Sonia Estrada Melgarejo (pintora peruana, Ancash) 


\section{RESUMEN}

Las fronteras entre países, representan cambios radicales en cuanto a costumbres, usos, conductas y normas, tales elementos tienden a individualizar al ser humano en su hacer diario, el tránsito de infantes de un país a otro es un hecho que siempre ha existido pero que a la fecha ha incrementado, haciendo relevante el estudio de esta conducta desde una perspectiva jurídico-social, enfocados en la denominación de estos sujetos por su calidad pero que al mismo tiempo analice al derecho como una fuente sustancial que rompe las barreras de territorio sobre los derechos universales denominado: Derechos Humanos. Este artículo examina los derechos humanos de las niñas, niños y adolescentes migrantes, el trato que reciben por parte de los Estados donde transitan, específicamente en los pasos fronterizos de México.

Palabras clave: derecho de menores, bienestar, migrantes, derechos humanos, fronteras.

\section{ABSTRACT}

The borders between countries, represent radical changes in customs, uses, behaviors and norms, such elements tend to individualize the human being in his daily work, the transit of childrens from one country to another is a fact that has always existed but recently has increased, making the study of this behavior relevant from a legal-social perspective, focused on the denomination of these subjects for their quality but at the same time analyzing the law as a substantial source that breaks down the barriers of territory over the Universal rights called: Human Rights. This article examines the human rights of migrant children and adolescents, the treatment they receive from the states where they travel, specifically at the Mexican border crossings.

Keywords: minors Rights, public policies, welfare, migrant, human rights. 
"La injusticia, en cualquier parte, es una amenaza a la justicia en todas partes."

Martin Luther King.

\section{INTRODUCCIÓN}

Por una parte, es menester establecer que la infancia, al ser distinta al adulto en su forma de actuar, pensar y sentir - y que dicha distinción es una característica estructural del desarrollo, por tanto, es una condición inmodificable en el sujeto a través de los primeros años de vida —, necesita un trato diferenciado. Por otra parte, es necesario reconocer que esto incrementa el grado y alcance de la obligación del Estado frente al propio derecho del niño, la niña o el adolescente. El conjunto de estas características colocan a la infancia en situación de especial vulnerabilidad con relación a la integridad de sus derechos. Cuando "se ven involucrados niños, niñas y adolescentes en situaciones migratorias irregulares, los ubica en una situación de vulnerabilidad especial que los hace susceptibles a padecer algunas formas de violencia ${ }^{1}$ ". Al respecto, la Corte Interamericana de Derechos Humanos ha sido constante en señalar que ante esta particular vulnerabilidad, el Estado tiene la obligación de prestar especial atención y asumir con mayor cuidado y responsabilidad su posición especial de garante.

El presente artículo estudia los derechos humanos de las niñas, niños y adolescentesen tránsito de un país a otro, enfatizando en los principios rectores de estos derechos que deben seguirles a cualquier lugar al que se trasladen, indiferentemente de su situación migratoria. Al respecto, los Estados deben actuar con mayor cautela y ejecutar medidas dirigidas a la protección de los derechos para esta clase vulnerable, por su condición de seres humanos, menores de edad, y por su calidad de migrantes.

1. Corte Interamericana de Derechos Humanos y Fondo de las Naciones Unidas para la Infancia, Violencias contra niñas, niños y adolescentes en América Latina y el Caribe, (San José: Corte Interamericana de Derechos Humanos y Fondo de las Naciones Unidas para la Infancia, 2019), 58. 
La migración infantil es un fenómeno que aumenta cada día y a la par también aumenta el número de violaciones a derechos humanos, esto se debe al contacto directo entre las niñas, niños y adolescentes y diversas instituciones, desde el momento en que ingresan a un territorio al que por nacionalidad no pertenecen, el trato que reciben durante su estadía y cuando son repatriados debe ser compatible con ponderar sus derechos fundamentales aun tratándose de migrantes ilegales. Las faltas recurrentes y que violentan los derechos de los menores, se debe al personal de las instituciones que atienden a estos menores, por lo que es apremiante que sean debidamente capacitados en cuanto a las normas universales y locales, ya que se trata de un grupo de ineludible protección jurídica por parte de los Estados.

Estos casos evidencian el mayor riesgo para las niñas, niños y adolescentes en estas situaciones, proveniente de las propias autoridades encargadas de ejercer el control migratorio. Independientemente de si los niños y niñas se encuentran acompañados por un familiar o están solos, la afectación más paradigmática consiste en el desconocimiento de sus derechos procesales en el trámite que permita identificar los motivos de su migración, su posible reconocimiento como refugiados y la posibilidad de solicitar asilo ${ }^{2}$.

Es importante responder a las necesidades más apremiantes de una población, las cuales serán consideradas de acuerdo a los objetivos para el desarrollo, las necesidades de salvaguardar la integridad física y psicológica de los menores de edad contribuyen a determinar la demanda de este grupo vulnerable, someterlas a metas internacionales, nacionales y estatales de la integración real del derecho, políticas públicas que deben ser formuladas en vías de desarrollo sustentable y eficaz, las condiciones actuales sobre el interés superior del menor de edad, han cambiado el paradigma de las relaciones interpersonales, sin embargo, también nos han obligado a enfrentarnos a nuevas conductas o condiciones de los menores de edad, estas conductas y condiciones se deben analizar y ponderar en el ejercicio de la potestad del Estado como ente regulador, y cumpliendo con su obligación de garantizar el catálogo de derechos que ostenta el grupo vulnerable identificado como menor de edad.

Partir del derecho de menores de edad en el mundo jurídico es caracterizar por las peculiaridades del sujeto al cual refiere, gozando de un derecho autónomo con la pronunciación de diversas normas internacionales, constitucionales, generales y las emanadas en los diversos Estados de nuestro país México, estas normas de aplicación en un procedimiento propio e indiferenciado, los anteriores calificativos o características que tienen que ser aplicados por jueces o tribunales especializados, actualmente se carece del ejercicio debido de este derecho discrecional en el desarrollo de derecho de los menores de edad por una gran parte de funcionarios que dudan de la autonomía científica de tal derecho.

2. Ídem. 
Para tales fines es necesario contar con investigaciones de calidad y cantidad, que evidencien la importancia de dar diagnósticos eficientes en seguridad y justicia que cada estado tiene que proporcionar a sus gobernados.

En esa tesitura, el presente artículo inicia con la exposición de la situación en las fronteras migratorias más transcurridas a nivel mundial, la frontera Sur y la frontera Norte de México, posteriormente se explica el reconocimiento de nuestro grupo en estudio, como sujetos de derechos, partiendo del análisis de la Convención sobre los Derechos del Niño, dentro del cual se establecen principios fundamentales para garantizar cabal protección a los derechos de las niñas, niños y adolescentes, por último, se presenta un análisis de las políticas públicas para protección del infante en la frontera.

\section{EL INFANTE COMO MIGRANTE Y LAS FRONTERAS DE MÉXICO}

La migración es entendida como el movimiento de personas de un Estado a otro con el propósito de radicarse de manera temporal y permanente, es un fenómeno cada vez más importante en las Américas $^{3}$, por si solo es un tema de interés, pero si hablamos de migración infantil debemos entender el alto grado de vulnerabilidad que corresponde a este grupo por su situación, máxime si se trata de menores que viajan o transitan no acompañados.

La Unidad de Política Migratoria ${ }^{4}$ muestra que de enero a septiembre de 2019 se tiene el registro de un total de 55,444 Niñas, Niños y Adolescentes migrantes, en este sentido se informa que en el 2019; 18,056 niños viajaban solos, estas cifras corresponden a los niños migrantes de nacionalidad mexicana que son repatriados desde los Estados Unidos y de aquellos de distintos países que cruzan a territorio mexicano, pues cada país tiene una delimitación geográfica, la cual sirve para separar una región de otra, asimismo cada uno con diferentes colindancias, que las identificamos como fronteras, por ejemplo, el territorio mexicano está integrado por entidades federativas; islas, arrecifes y cayos en los mares adyacentes; las islas Guadalupe y las de Revillagigedo situadas en el Océano Pacífico; la plataforma continental y los zócalos submarinos; las aguas de los mares territoriales y los mares interiores, y el espacio aéreo situado sobre el territorio nacional ${ }^{5}$ pero en general, se compone de cuatro fronteras, mejor conocidas por su orientación geográfica, dos de ellas menos conocidas y transitadas, estas son las fronteras Este y Oeste, mientras que las Fronteras Norte y Sur son conocidas por el tránsito de personas, que en su mayoría lo hacen de manera ilegal.

3. Helena Olea, et al., El Sistema Interamericano de Protección de los Derechos Humanos y los derechos de las poblaciones migrantes, las mujeres, los pueblos indígenas y los niños, niñas y adolescentes, (San José: Instituto Interamericano de Derechos Humanos, 2004), 13.

4. Véase http://www.politicamigratoria.gob.mx

5. Véase artículo 42 de la Constitución Política de los Estados Unidos Mexicanos. 


\subsection{La frontera sur (Guatemala y Belice)}

Corresponde a la limitación geográfica que existe entre el territorio mexicano y los países de Guatemala y Belice, no es una novedad que por esta ruta exista el tránsito de migrantes pero en los últimos años se ha incrementado, para ello basta recordar las "caravanas migrantes", en tanto que "la presencia de caravanas que transitan por México tampoco representa una realidad nueva, ya que desde 2011 se han desarrollado de modo continuo por el país ${ }^{6}$, en estos últimos ańos han tomado mayor fuerza al tratarse de miles de inmigrantes indocumentados que ingresan al país, algunos con las esperanzas de llegar a los EE. UU., y otros para establecerse en México, lo que nos es reafirmado por Ahedo de la Reguera al manifestar que "la mayor parte de las personas que migran al territorio mexicano, transitan por esta frontera únicamente porconexión para llegar a los Estados Unidos de Norteamérica”7

\subsection{La frontera norte (Estados Unidos de Norteamérica)}

Es de todas, la más importante, aunque solamente colinda con un país, ya que se trata de uno de los países más importantes del mundo. Considerada como una de las fronteras más transitadas del mundo, la meta es llegar al territorio de los EEUU para cumplir el "sueño americano", viajan desde el exterior de la República mexicana hasta los del interior. Cabe resaltar que los Estados que colindan con Estados Unidos tienen estadísticamente el mayor número de niñas, niños y adolescentes repatriados a México.

\section{LAS NIÑAS, NIÑOS Y ADOLESCENTES COMO SUJETOS DE DERECHOS}

La Doctrina de la Protección Integral, cuyo fundamento jurídico-filosófico surge de un conjunto de instrumentos internacionales en materia de derechos humanos que tienen como máximo exponente la Convención sobre los Derechos del Niño, abre paso a una revolución pacífica que lucha por el reconocimiento de los derechos de niñas, niños y adolescentes sobre la premisa fundamental de la confirmación de su estatus jurídico como sujetos de derecho.

El concepto niñez incluye a aquellas personas que requieren de determinadas medidas o cuidados especiales por su situación de vulnerabilidad en que se encuentran frente al ordenamiento jurídico ${ }^{8}$.

6. Alethia Fernández de la Reguera Ahedo, et al., Caravanas migrantes: las respuestas de México. Serie Opiniones Técnicas sobre Temas de Relevancia Nacional, (México: Universidad Nacional Autónoma de México, 2019), 44.

7. Ibídem, 45

8 En un criterio reciente, se aseveró que la protección constitucional que merecen los niños no se equipara a la que debe recibir cualquier otro grupo vulnerable. La mayor protección a sus derechos no sólo se justifica por su situación de mayor vulnerabilidad, sino también por el interés específico de la sociedad en velar porque los menores alcancen su pleno desarrollo. En este sentido, el principio del interés superior ordena a todas las autoridades estatales que la protección de los derechos del niño se realice a través de medidas "reforzadas" o "agravadas", y que los intereses de los niños sean protegidos con mayor contundencia. Cfr. Tesis 1a. CCC/2015, Décima Época, Primera Sala, Semanario Judicial de la Federación, 09 de octubre de 2015, bajo el rubro: BULLYING ESCOLAR. EXISTE UN DEBER DE DEBIDA DILIGENCIA DEL ESTADO PARA PROTEGER Y GARANTIZAR LOS DERECHOS DE LOS MENORES DE ACUERDO CON EL 
El Poder Judicial de la Federación avala este nuevo criterio al aplicar una interpretación sistemática y funcional de los artículos 1o. de la Constitución Política de los Estados Unidos Mexicanos (CPEUM), así como de los artículos 1 y 12 de la Convención Americana sobre Derechos Humanos, de donde se deriva el igual reconocimiento que estos organismos internacionales aplican a las personas con discapacidad ante la ley y la obligación del Estado para adoptar medidas pertinentes que puedan ejercer plenamente su capacidad jurídica.

En este sentido, la aparente vaguedad de los términos empleados obedecía a que ninguno de los dos cuerpos normativos antes citados constituye un instrumento especializado en la protección de los derechos de la nińez; sin embargo, ambos reconocen la importancia de establecer expresamente una fórmula que dé entrada a los distintos derechos o medidas previstas en los ordenamientos que sí se especializan en la materia; de ahí que la falta de una regulación específica del catálogo de los derechos que conformaban el corpus iuris de protección constitucional y convencional de la niñez no implicaba su desconocimiento, sino por el contrario, remitía a los instrumentos que en forma especializada cumplen con dicha misión. Consecuentemente, el citado artículo 4o., párrafo octavo, de la CPEUM, representaba un punto de convergencia con los derechos de los menores de edad reconocidos en tratados internacionales y constituía un parámetro de regularidad especializado respecto de los derechos de la niñez, como el que establece el artículo 1o., párrafo segundo de la Constitución mexicana respecto de los derechos humanos en general.

El proceso de reconocimiento jurídico de los derechos del niño fue en forma paulatina a través de numerosos y trascendentes pactos internacionales relativos a los derechos de la humanidad que incluían al niño en forma más o menos directa, hasta que se hizo necesario otorgar un instrumento que reflejara tales avances, y que a la vez constituyera un compromiso de la sociedad, representada por los distintos Estados, respecto de considerar al niño en la plenitud de su proyección como persona, lo que finalmente se concretó en una Convención sobre los derechos del niño 9 .

\subsection{La Convención sobre los Derechos del Nińo (CDN)}

La protección a los derechos de este grupo no se remite únicamente a este instrumento internacional, pues la protección a los Derechos de las niñas, niños y adolescentes, se debe primeramente a su condición de seres humanos, por lo que su protección inicia con la Declaración Universal de los Derechos Humanos, así como por la Convención Americana de los Derechos Humanos.

Este tratado internacional, es caracterizado por ser el primero en dirigirse específicamente a los derechos de las niñas, niños y adolescentes del mundo, fue firmado por 20 países el 20 de noviembre de 1989, con el transcurso de los años se fueron integrando más países, su aplicación es obligatoria en los

PRINCIPIO DE SU INTERÉS SUPERIOR.

9. Marissa A. Jaggli, Menores en riesgo social, (Córdoba: Mediterránea, 2007), 21. 
países que la reconocen, consta de 54 artículos, que incluyen derechos económicos, sociales, civiles, políticos y culturales. El Estado mexicanolo ratificó el 21 de septiembre de 1990, dicho instrumento prevé, entre otros, el acceso a un desarrollo pleno, a la protección contra influencias peligrosas, los malos tratos y la explotación, así como a la plena participación en la vida familiar, cultural y social. Este instrumento internacional reconoce los diversos derechos a las niñas, niños y adolescentes, en esta ocasión enunciaré aquellos que tienen relación con nuestro objeto en estudio:

A la vida, artículo 6;

A la identidad, articulo 7 y 8 ;

A una familia, articulo 9;

A opinar, artículo 12 y 13;

A protección y cuidado, artículos 1, 2, 3, 4, 18, 19, 20, 23, 32, 33, 34, 35, 36, 37, 38, 39 y 40;

A salir de cualquier país, articulo 10;

A ser escuchado, artículo 12;

A la información nacional y extranjera, articulo 17;

A la salud, artículos 1, 2, 3, 4, 24 y 25;

A seguridad social, artículos 2, 26 y 39;

A un nivel de vida adecuado, artículo 1, 2, 3, 4y 27; y

A la educación, artículo 1, 2, 3, 28, 29 y 30.

"La Convención resulta entonces el marco mínimo de reconocimiento y respeto a los derechos de los niños en el que deben inscribirse las prácticas y las políticas de los países que la han suscripto ${ }^{10}$ ”. Vale la pena subrayar que esta convención no solo es importante por definir los derechos humanos de las niñas y niños, sino también por ser el primer instrumento internacional jurídicamente obligatorio para los Estados que la han ratificado, esto quiere decir que sin importar la nacionalidad del infante, ni su estatus migratorio, cuando alguno se encuentre en otro territorio que haya ratificado la CDN se verá protegido jurídicamente.

\subsection{Principios aplicables al infante}

Dentro de la normativa nacional e internacional, se ha evolucionado en la concepción jurídica que se tiene sobre los menores, sus derechos y su salvaguarda, así como en los criterios orientadores. El

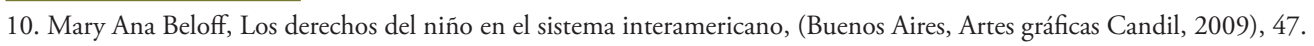


Comité de los Derechos del Niño, estableció en su primer periodo de sesiones en 1991, los principios generales de la Convención:

La no discriminación (artículo 2);

El interés superior del niño (artículo 3);

El derecho a la vida, supervivencia y desarrollo (artículo 6); y

El respeto a la opinión del niño (artículo 12).

Estos son principios propios esencialmente diferentes a los que norman el derecho en general y al mismo tiempo son principios rectores que constituyen el fundamento de los derechos de los niños y niñas ${ }^{11}$.

\subsubsection{No discriminación}

Es un principio fundamental, que se encuentra establecido en los diversos instrumentos internacionales, es considerado por la Corte un "principio básico y general de la protección de los derechos humanos, alcanzando el carácter de iuscogens, su importancia es de la misma magnitud en el derecho interno y se trata de un principio de derecho imperativo ${ }^{12}$ ". El Comité de Derechos Humanos ha definido la discriminación como:

Toda distinción, exclusión, restricción o preferencia que se basen en determinados motivos, como la raza, el color, el sexo, el idioma, la religión, la opinión política o de otra índole, el origen nacional o social, la propiedad, el nacimiento o cualquier otra condición social, y que tengan por objeto o por resultado anular o menoscabar el reconocimiento, goce o ejercicio, en condiciones de igualdad, de los derechos humanos y libertades fundamentales de todas las personas.

Por pronunciamientos de la Corte, la no discriminación, siempre es enlazada con la igualdad, y los considera "elementos constitutivos de un principio básico y general relacionado con la protección de los derechos humanos"13.

Desde mi punto de vista considero que la igualdad vendría a ser un efecto de la aplicación correcta de este principio (no discriminación), de manera tal que si no hay discriminación tenemos igualdad ante la ley, logrando que de esta manera se de cumplimiento efectivo a la protección de la ley a favor de todas las personas.

11. María Delgadina Valenzuela Reyes, Derechos Humanos de los niños y las niñas. (México: Porrúa, 2013), 217.

12. Corte Interamericana de Derechos Humanos, Cuadernillo de Jurisprudencia de la Corte Interamericana de Derechos Humanos No. 14 : Igualdad y no discriminación, (San José: : Corte IDH, 2019), 11, acceso el 15 de enero de 2020, http:// www.corteidh.or.cr/sitios/libros/todos/docs/cuadernillo14.pdf

13. Ibídem, 6 . 
Específicamente a la norma internacional que opera respecto a nuestro grupo en estudio este principio se reconoce en el artículo 2 párrafo primero de la CDN, en el que señala que los derechos enunciados en ella, serán aplicados por los Estados partes a cada nińo sujeto a su jurisdicción, sin distinción alguna, sea por raza, color, sexo, idioma, religión, opinión política o de otra índole, y fundamental resaltar que expresa literalmente que dichas distinciones tampoco serán aplicadas aún sin importar el origen nacional, étnico o social, la posición económica, los impedimentos físicos, el nacimiento o cualquier otra condición del niño, de sus padres o de sus representantes legales. Es así que si de los niños migrantes se habla, los estados parte de esta Convención no deben visualizar características de discriminación como las mencionadas para asegurar a estos menores de edad el disfrute a todos los demás derechos enunciativos en dicho instrumento. Dentro de nuestra norma Constitucional, en cuanto a lo que nos ocupa, en su primer artículo nos señala que "esta prohibida toda discriminación motivada por origen étnico o nacional, el género, la edad, [...] que atente contra la dignidad humana y tenga por objeto anular o menoscabar los derechos y libertades de las personas".

En este sentido, los Estados dan cumplimiento a este principio al no introducir en su ordenamiento jurídico regulaciones discriminatorias, pero ese no es todo el alcance de este principio sino que debe ser a tal grado que se logre eliminar las regulaciones de carácter discriminatorio y en ese actuar combatir las prácticas de este carácter, estableciendo normas y medidas que reconozcan y aseguren la efectiva igualdad ante la ley de todas las personas ${ }^{14}$.

\subsubsection{El interés superior del menor}

En México, la Primera Sala de la Suprema Corte de Justicia de la Nación (SCJN) ${ }^{15}$ ha sostenido que el interés superior de la infancia, asentado expresamente en el artículo 4o., párrafo octavo de la CPEUM, a partir de la reforma constitucional de 12 de octubre de 2011, exige la "garantía plena" de los derechos de las niñas y los niños. Cabe resaltar que aun cuando el significado de la expresión "los derechos" puede parecer vaga, el texto del que deriva es similar al del artículo 19 de la Convención Americana sobre Derechos Humanos, específicamente en la fracción que reconoce el derecho de los menores de edad a ciertas "medidas de protección" que, al igual que en la Constitución General de la República, no se enuncian puntualmente.

El interés superior del niño es un principio transversal que define en gran medida el alcance y obligación del Estado frente a la infancia. Dicho principio significa que se privilegie los derechos del niño por sobre los derechos de los adultos, lo cual sería insostenible, ya que ningún derecho puede por sí mismo ser más valioso que otro, así como ningún ser humano puede ser más valioso que otro.

\section{Idem.}

15. Tesis 1a. LXXVI/2013, Décima Época, Primera Sala, Semanario Judicial de la Federación y su Gaceta, Libro XVIII, marzo de 2013, t. 1, p. 887; bajo el rubro: INTERÉS SUPERIOR DE LA NIÑEZ. EL ARTÍCULO 4O. PÁRRAFO OCTAVO, DE LA CONSTITUCIÓN POLÍTICA DE LOS ESTADOS UNIDOS MEXICANOS, REPRESENTA UN PUNTO DE CONVERGENCIA CON LOS DERECHOS DE LA INFANCIA RECONOCIDOS EN TRATADOS INTERNACIONALES. 
El principio del interés superior del niño es más bien una herramienta hermenéutica para definir la obligación del Estado y cómo se debe actuar frente a la infancia. Se constituye como eje rector que gobierna toda acción pública que afecta al niño y a la niña. En este sentido, el mismo principio trata más de la obligación del Estado que del derecho del niño.

En ese contexto, hoy nuestra legislación resalta de distintas formas la protección del Principio del Interés Superior del Menor, palabra que se ha vuelto el argumento y fundamento más sólido sobre el cual reposa el objeto en muchos casos de los juicios de Controversia sobre el Estado Civil de las Personas y del Derecho Familiar o de Familia.

En esa tesitura, la evolución del pensamiento jurídico actual permite hacer alusión al término interés superior del menor, "[...] segmento de personas que tienen entre cero y dieciocho años incompletos, a las que dependiendo el rango de edad se les denomina genéricamente niños" ${ }^{16}$ quienes gozan de los derechos consagrados para los seres humanos y que es deber de los Estados promover y garantizar su efectiva protección igualitaria.

La Corte Interamericana de Derechos Humanos en su opinión consultiva OC-17/2002 de fecha 28 de agosto de 2002, solicitada por la Comisión Interamericana de Derechos define el interés superior del niño en los párrafos 56 al 61, donde describe que este principio regulador de la normativa de los derechos del niño se funda en la dignidad misma del ser humano, en las características propias de los niños, y en la necesidad de propiciar el desarrollo de éstos, con pleno aprovechamiento de sus potencialidades así como en la naturaleza y alcances de la Convención sobre los Derechos del Niño.

A continuación vincula la Corte el principio con la CDN [que] alude al interés superior de éste (artículos 3, 9, 18, 20, 21, 37 y 40) como punto de referencia para asegurar la efectiva realización de todos los derechos contemplados en ese instrumento, cuya observancia permitirá al sujeto el más amplio desenvolvimiento de sus potencialidades. A este criterio han de ceñirse las acciones del Estado y de la sociedad en lo que respecta a la protección de los niños y a la promoción y preservación de sus derechos."

Asimismo, la Corte Interamericana ha resuelto en diversos casos que la condición de garante del Estado con respecto a este derecho, le obliga a prevenir situaciones que pudieran conducir, por acción u omisión, a la afectación de aquél ${ }^{17}$. La Corte IDH establece de manera clara que en esta materia, cuando se trata de la protección de los derechos del niño y de la adopción de medidas para lograr dicha protección, rige el principio del interés superior del niño ${ }^{18}$. En su decisión sobre el caso de las niñas Yean y Bosico contra República Dominicana, la Corte afirmó que "la prevalencia del interés superior del niño debe ser entendida como la necesidad de satisfacción de todos los derechos de los menores, que obliga al Estado e irradia efectos en la interpretación de todos los demás derechos de

16. Mauricio González Oviedo, Derechos de la niñez y la adolescencia, (Costa Rica: Comisión Nacional para el Mejoramiento de la Administración de Justicia, 2001) 35, acceso 15 de enero de 2020, https://www.unicef.org/costarica/docs/cr_pub_Antologia_derechos_NNA_Escuela_Judicial.pdf

17. Corte I.D.H., Caso de la masacre de Mapiripán vs. Colombia... párr. 162.

18. Corte I.D.H.: Caso Bulacio vs. Argentina. Sentencia de 18 de septiembre de 2003. Serie C No 100, par. 134, 
la Convención cuando el caso se refiera a menores de edad ${ }^{19}$ ”. En particular, el Principio del Interés Superior de la Niñez significa que:

1) Toda acción pública debe basar su determinación de qué es lo mejor para el niño y la niña en los propios derechos del niño como parámetro. Ninguna acción deberá ser discrecional y basada en un criterio arbitrario sobre qué es lo más conveniente para el niño, la niña o el adolescente.

2) Toda acción pública con relación a la infancia debe basarse en una valoración integral del conjunto de los derechos del niño(a) y estos mismos proyectados hacia el futuro.

3) El Estado tiene frente a la infancia una obligación reforzada.

La primera implicación pudiera incluso parecer ociosa al establecer algo que desde una perspectiva de derechos resulta obvio. Sin embargo, dado que históricamente las acciones públicas hacia la infancia se han caracterizado por el paternalismo o visiones tutelares, dicha implicación se torna indispensable. El único parámetro para la determinación del interés superior del niño lo constituye el ejercicio integral de sus derechos.

Consecuentemente, la segunda implicación es de enorme relevancia, porque el carácter integral de los derechos de la infancia es un elemento estrechamente vinculado con la propia condición del ser humano en sus primeros años de desarrollo. En este sentido, se construye a partir de las necesidades del niño y la niña, y coloca el ejercicio de todos y cada uno de sus derechos como requisito para el sano desarrollo. Esto conlleva significativas implicaciones para el alcance de la obligación del Estado y establece la pauta de dicha obligación basada en la necesidad del nińo y la nińa, y no así en la facultad o capacidad de un actor público. Tiene implicaciones importantes en cuanto al grado de acción oficiosa que se hace exigible a todo servidor público, al grado de establecer obligaciones adicionales a las estipuladas en las facultades concedidas por ley para dar vista y accionar todo aquello necesario para el resguardo integral de los derechos de un niño o niña.

Finalmente, el conjunto de particularidades que definen la obligación del Estado frente a la infancia redundan en la tercera gran implicación del principio del interés superior del niño: el carácter reforzado de la obligación del Estado.

El texto actual del artículo $4^{20}$ constitucional, en la parte que regula el interés superior de la infancia, es el resultado de la reforma publicada en el Diario Oficial de la Federación el 12 de octubre de

19. Corte I.D.H.: Caso de las Niñas Yean y Bosico vs. República Dominicana. Excepciones Preliminares, Fondo, Reparaciones y Costas. Sentencia de 8 de septiembre de 2005. Serie C No 130, par. 134, 59; Cfr. Corte I.D.H.: Condición Jurídica y Derechos Humanos del Niño. Opinión Consultiva OC-17/02 de 28 de agosto de 2002. Serie A Nº 17, pars. 56, 57 y 60.

20. Artículo $4^{\circ}$. [...]. En todas las decisiones y actuaciones del Estado se velará y cumplirá con el principio del interés superior de la nińez, garantizando de manera plena sus derechos. Los niños y las niñas tienen derecho a la satisfacción de sus necesidades de alimentación, salud, educación y sano esparcimiento para su desarrollo integral. Este principio deberá guiar el diseño, ejecución, seguimiento y evaluación de las políticas públicas dirigidas a la niñez.

Los ascendientes, tutores y custodios tienen la obligación de preservar y exigir el cumplimiento de estos derechos y principios.

El Estado otorgará facilidades a los particulares para que coadyuven al cumplimiento de los derechos de la niñez. $[\ldots]$. 
2011. No obstante, el interés superior de la niñez ya era reconocido como un principio constitucional implícito, además de que se encontraba tutelado por los tratados internacionales que son parte del ordenamiento jurídico mexicano, consecuentemente, su observancia era una obligación de los juzgadores nacionales.

Desde hace algunos años, la Primera Sala de la SCJN conceptualizó el interés superior de la niñez como un principio -de naturaleza constitucional e internacional- que considera el desarrollo de las niñas, niños o adolescentes, el ejercicio pleno de sus derechos como criterios rectores en la elaboración de normas y su aplicación en todos los órdenes relativos a la vida del niño. ${ }^{21}$

El interés superior de la niñez cumple con varias dimensiones o funciones normativas ${ }^{22}$ : (i) como pauta interpretativa aplicable a las normas y actos que tengan injerencias respecto de los derechos de niñas, niños o adolescentes ${ }^{23}$; y (ii) como principio jurídico rector que exige una máxima e integral protección de los derechos cuya titularidad corresponde a un menor de edad. ${ }^{24}$

Esta función normativa también impone una doble carga para las autoridades jurisdiccionales que se encarguen de velar por el interés superior de la niñez. En primer lugar, dichas autoridades deben interpretar sistemáticamente todo el entramado normativo que protege los derechos de la infancia. En segundo lugar, se impone una carga a los juzgadores para que lleven a cabo un escrutinio mucho más estricto de la necesidad y proporcionalidad de las medidas que puedan afectar los derechos de las niñas, niños o adolescentes. ${ }^{25}$

21. Tesis aislada 1a. CXLI/2007, registro de IUS 172003, publicada en el Semanario Judicial de la Federación y su Gaceta, Novena Época, tomo XXVI, julio de 2007, página 265, cuyo rubro es "INTERÉS SUPERIOR DEL NIÑO. SU CONCEPTO”.

22. Tesis aislada 1a. CXXI/2012 (10a.), registro de IUS 2000989, publicada en el Semanario Judicial de la Federación y su Gaceta, Décima Época, Libro IX, junio de 2012, Tomo 1, página 261, cuyo rubro es "INTERÉS SUPERIOR DEL MENOR. SUS ALCANCES Y FUNCIONES NORMATIVAS".

23. Tesis aislada 1a. CXXIII/2012 (10a.), registro de IUS 2000987, publicada en el Semanario Judicial de la Federación y su Gaceta, Décima Época, Libro IX, junio de 2012, Tomo 1, página 259, cuyo rubro es "INTERÉS SUPERIOR DEL MENOR. SU FUNCIÓN NORMATIVA COMO PAUTA INTERPRETATIVA PARA SOLUCIONAR CONFLICTOS POR INCOMPATIBILIDAD EN EL EJERCICIO CONJUNTO DE LOS DERECHOS DE LOS NIÑOS”.

24. Tesis aislada 1a. CXXII/2012 (10a.), registro de IUS 2000988, publicada en el Semanario Judicial de la Federación y su Gaceta, Décima Época, Libro IX, junio de 2012, Tomo 1, página 260, cuyo rubro es "INTERÉS SUPERIOR DEL MENOR. SU FUNCIÓN NORMATIVA COMO PRINCIPIO JURÍDICO PROTECTOR”. Ver, en un sentido similar, la tesis aislada P. XLV/2008, registro de IUS 169457, publicada en el Semanario Judicial de la Federación y su Gaceta, Novena Época, tomo XXVII, junio de 2008, página 712, cuyo rubro es "MENORES DE DIECIOCHO AÑOS. EL ANÁLISIS DE UNA REGULACIÓN RESPECTO DE ELLOS DEBE HACERSE ATENDIENDO AL INTERÉS SUPERIOR Y A LA PRIORIDAD DE LA INFANCIA".

25. Tesis aislada 1a. XV/2011, registro de IUS 162807, publicada en el Semanario Judicial de la Federación y su Gaceta, Novena Época, XXXIII, febrero de 2011, página 616, cuyo rubro es "INTERÉS SUPERIOR DEL NINOO. FUNCIÓN EN EL AMBITO JURISDICCIONAL". 
Ahora bien, con motivo de la reforma al artículo $4^{\circ}$ constitucional de 7 de abril de 2000, la Primera Sala de la SCJN reconoció el interés superior de la infancia como un principio implícito de rango constitucional y como un punto de convergencia con el corpus iuris internacional de protección de la niñez. ${ }^{26}$ No obstante, como se mencionó anteriormente, la reforma del 12 de octubre de 2011 reconoció expresamente el interés superior de la niñez como principio constitucional.

Así, la regulación constitucional del derecho de los menores de edad a participar en los asuntos que puedan afectar su esfera jurídica parte del interés superior de la infancia.

\subsubsection{Vida, supervivencia y desarrollo}

Los derechos de supervivencia, son los de necesidades fundamentales para todo ser humano, contribuyen al desarrollo de la personalidad y la promoción de la dignidad humana. Esto derechos hacen reflexión sobre la condición de dignidad, la cual es vista como la posibilidad que tiene todo ciudadano a vivir en un contexto armonioso, en donde su integridad no se encuentre en riesgo ni en su dimensión física ni en su dimensión mental ${ }^{27}$. En estos se encuentran inmersos diversos derechos de supervivencia, para esta ocasión los delimitaremos en derecho a la salud, a la nutrición y a la seguridad social.

El Derecho a la Salud, está reconocido a través de la CDN en los artículos 1, 2, 3, 4, 24.1 y 25 y en el artículo $4^{28}$ de la Constitución mexicana, que se refiere a que los menores de edad tienen derecho al disfrute del más alto nivel de salud, entendiendo que este se refiere a un "estado de completo bienestar físico, mental y social, en tanto se deduce que la falta de alguno determina que un individuo carece de esta" ${ }^{29}$

El Derecho de alimentación, no se encuentra explícitamente en la CDN, sino que lo integra como parte del derecho a la salud, de manera que "los medios para tener acceso a ellos son las condiciones

26. En el amparo directo en revisión 1187/2010, resuelto el $1^{\circ}$ de septiembre de 2010 por unanimidad de 5 votos, se reconoció esta situación. Asimismo, en dicho asunto se mencionó que en el dictamen sobre la iniciativa de reforma al artículo $4^{\mathrm{o}}$ constitucional de las Comisiones Unidas de Puntos Constitucionales, de atención a Niños Jóvenes y Tercer Edad y de Estudios Legislativos de la Cámara de Senadores, de 9 de diciembre de 1999, se sostuvo que "no escapa a estas Comisiones Unidas el hecho de que resulta necesario para la citada reforma constitucional reconocer ideales consignados en la legislación internacional, así como los generados en diversos foros en la materia”. Por su parte, en el dictamen de la Comisión de Gobernación y Puntos Constitucionales de la Cámara de Diputados, que actuó como cámara revisora de aquella iniciativa de reforma, de 15 de diciembre de 1999, se resalta "la pertinencia de actualizar el contenido del vigente párrafo final del artículo cuarto constitucional, a la luz de los compromisos internacionales suscritos por nuestro país respecto de los derechos de niños y de niñas”. Véase tesis aislada 1a. XLVII/2011, registro de IUS 162354, publicada en el Semanario Judicial de la Federación y su Gaceta, Novena Época, tomo XXXIII, abril de 2011, página 310, "INTERÉS SUPERIOR DEL NIÑO. ES UN PRINCIPIO DE RANGO CONSTITUCIONAL IMPLÍCITO EN LA REGULACIÓN DE LOS DERECHOS DE LOS MENORES PREVISTOS EN EL ARTÍCULO 4o. CONSTITUCIONAL".

27. Nidia Ximena Mancera Mahecha, et. al., derechos de supervivencia: fundamentos para una vida digna, Hojas y hablas, 10 (2013): 110.

28. Toda persona tiene derecho a la protección de la salud.

29. Rolando Castillo Santiago, "La alienación parental: conducta que atenta la salud", en La Convención sobre los derechos del niño a debate 30 años después, ed. Por Antonio Salvador Jiménez Hernández (México: CIPI Infancia, 2019) 534535. 
que brindan los Estados para que todos los ciudadanos tengan acceso a una adecuada alimentación y también como las posibilidades de independencia económica para conseguirlos"30.

El Derecho a la seguridad social se expresa en los artículo 2, 26.1 y 39 de la CDN, la finalidad de este "va más allá del derecho a la salud, pues comprende asistencia médica, la protección de los medios de subsistencia y los servicios sociales necesarios para el bienestar individual y colectivo, incluyendo en su caso el otorgamiento de una pensión" ${ }^{31}$

Por su parte el Comité de los Derechos del Niño de la ONU concluye que "el desarrollo del niño es un concepto holístico que abarca el desarrollo físico, mental, espiritual, moral, psicológico y social"32.

Lo antes mencionado se circunscribe de manera conjunta en este principio.

\subsubsection{Opinar y a ser oído}

El Comité de los Derechos del Niño, sustentó en su primer periodo de sesiones en 1991, que este es un principio general de la CDN, elevar el derecho a opinar a la categoría de principio tiene claras y precisas implicaciones. Cuando se habla de principios en un sistema jurídico basado en el reconocimiento de derechos, puede afirmarse que los principios son derechos que permiten a su vez ejercer otros y resolver conflictos entre derechos igualmente reconocidos. En este orden, para que el niño pueda ejercerlos efectivamente, debe necesariamente escuchar la opinión del menor sobre lo que le concierne y posteriormente a una valoración debe respetarse su interés en todas las circunstancias que puedan afectarlo, como por ejemplo: los casos donde se ven involucrados niños, niñas y adolescentes en situaciones migratorias irregulares, en los que "no suelen ser escuchados en estos procesos, desconociendo su derecho de participación en los procedimientos que los afectan, y con ello, además, obstaculizando la identificación de lo que en estas circunstancias representaría el interés superior para ellos”.

En consecuencia, la opinión del niño debe tomarse en cuenta al momento de interpretar y aplicar en forma práctica cada uno de los derechos que en ella se enuncian, convirtiéndose así en un imperativo para los Estados Partes adoptarlo en sus legislaciones internas, pues no es posible concebir una ley en materia de niños, que pretenda acoger la Doctrina de la Protección Integral y no consagre este derecho en toda su extensión.

El artículo 19 de la Declaración Universal de Derechos Humanos, estipula que "todo individuo tiene derecho a la libertad de opinión y expresión; este derecho incluye el no ser molestado a causa de sus opiniones, el de investigar y recibir informaciones y opiniones, y el de difundirlas, sin limitación de fronteras, por cualquier medio de expresión”. Por su parte, el Pacto Internacional de Derechos Civiles y Políticos en su artículo 19 dispone:

30. Idem, 112.

31. Joel Francisco Jiménez García, El derecho del menor, (México: Universidad Nacional Autónoma de México, 2012), 147-148.

32. Cfr. ONU. Comité de los Derechos del Niño, Observación General No. 5 de 27 de noviembre de 2003,párr. 
1. Nadie podrá ser molestado a causa de sus opiniones.

2. Toda persona tiene derecho a la libertad de expresión; este derecho comprende la libertad de buscar, recibir y difundir informaciones e ideas de toda índole, sin consideración de fronteras, ya sea oralmente, por escrito o en forma impresa, artística, o por cualquier otro procedimiento de su elección.

3. El ejercicio del derecho previsto en el párrafo 2 de este artículo entraña deberes y responsabilidades especiales. Por consiguiente puede estar sujeto a ciertas restricciones, que deberán, sin embargo, estar expresamente fijadas por la ley y ser necesarias para:

a) Asegurar el respeto a los derechos o a la reputación de los demás.

b) La protección de la seguridad nacional, el orden público o la salud o la moral públicas.

Dentro del Sistema Interamericano, la Declaración Americana sobre Derechos y Deberes del Hombre también pauta en su artículo 4 que "toda persona tiene derecho a la libertad de investigación, de opinión, de expresión y de difusión del pensamiento por cualquier medio”.

Si bien es cierto que sobre estas bases se reconoce como derecho humano el derecho que tiene toda persona a emitir sus opiniones, su consagración y regulación en la Convención sobre los Derechos del Niño debía responder a las particulares condiciones de crecimiento de los niños para ser acordes con los criterios de desarrollo humano que ha establecido la psicología evolutiva, y según la cual el individuo en cada una de las grandes etapas de su desarrollo antes de llegar a la vida adulta presenta características propias e individuales que merecen ser abordadas de manera diferente.

Aunado a lo anterior, la transformación de los nińos en verdaderos sujetos de derecho trae consigo no sólo que sean reconocidos como titulares de derechos y deberes, sino que de acuerdo con su desarrollo evolutivo se les otorgue la capacidad para ejercer personal y directamente sus derechos en forma progresiva y bajo este mismo criterio asuman deberes y responsabilidades por sus actos, asegurándose de esta manera su desarrollo integral, estimulando así la formación de adultos útiles, participativos e integrados a la sociedad, pero por sobre todas las cosas un individuo capaz de tomar sus propias decisiones en forma consciente y responsable. En definitiva el niño debe ser concebido como un sujeto activo de derechos.

Conjugando estos aspectos, el artículo 12 de la Convención sobre los Derechos del Niño regula el derecho a opinar de los niños en los siguientes términos:

1. Los Estados Partes garantizarán al niño que esté en condiciones de formarse un juicio propio, el derecho de expresar su opinión libremente en todos los asuntos que afectan al niño, teniéndose debidamente en cuenta las opiniones del niño, en función de la edad y madurez del niño. 
2. Con tal fin, se dará en particular al niño la oportunidad de ser escuchado en todo procedimiento judicial o administrativo que afecte al nińo, ya sea directamente o por medio de un representante o de un órgano apropiado, en consonancia con las normas de procedimiento de la ley nacional.

En primer lugar, el párrafo número uno (1) alude propiamente al contenido y alcance del derecho en estudio, el cual se proyecta hacia tres elementos claramente definidos en la norma, a saber: El derecho a expresar su opinión, el derecho a ser oído y el derecho a que tales opiniones sean debidamente tomadas en cuenta en razón de la edad y madurez del niño.

Y el párrafo número dos (2) especifica que el ejercicio de tal derecho debe extenderse a todo procedimiento administrativo o judicial donde se ventile algún asunto relacionado con el niño.

\section{CONCLUSIONES}

-Las niñas, niños y adolescentes, su individualización como grupo vulnerable se debe a su especificidad, es decir, menores de edad, considerados como seres especialmente débiles y quienes, si bien son protegidos por los Instrumentos Internacionales y las normas del Derecho interno, requieren que los Estados den cumplimiento a ellas a través de sus políticas públicas, y en el trato de los menores de edad migrante en su territorio.

-Todo actuar del Estado debe estar dirigido para que este grupo minoritario alcance el bienestar con todo lo que lo integra, esto sin importar el lugar de residencia, en virtud de que son sujetos de derechos por su condición de seres humanos y no por su edad o nacionalidad.

-Como se ha podido observar a lo largo del presente, existen los elementos necesarios para normar la aplicación del derecho alas niñas, niños o adolescentes en cualquier asunto que los afecte, en cumplimiento del interés superior del menor y de su derecho a ser tratado en cualquier proceso, administrativo o judicial en donde se encuentre inmerso, como puede tratarse en de cualquier proceso o tramite referente a su situación migratoria. A los Estados a través de sus operadores, sólo resta la voluntad y el cambio de paradigma mental, que dé cabida a la aplicación del Derecho con sus debidas suplencias, para lograr la participación auténtica de las niñas, niños y adolescentes en estos casos.

-Finalmente, todo lo abordado en el presente, se encuentra establecido en la síntesis que elaboró la Corte en colaboración con el Fondo de las Naciones Unidas para la Infancia:

\section{Sobre el proceso migratorio}

- Respeto a las garantías del debido proceso en los trámites migratorios, independientemente del carácter que tenga la autoridad: administrativo o judicial.

- Acceso a la comunicación con asesores legales, familiares o agentes consulares.

- La designación de un tutor o tutora es indispensable para iniciar cualquier proceso migratorio 
- donde se vea involucrado algún niño, niña o adolescente, y la asistencia consular es prioritaria.

- Los procesos deben realizarse de forma individual, considerando las particularidades de las personas sobre las cuales se decide su situación migratoria.

- Necesidad de motivación de las decisiones.

\section{Sobre la expulsión o deportación}

- Los procedimientos de expulsión o deportación solo puede realizarlos una autoridad competente.

- Procedimientos que deben adoptarse para determinar una expulsión o deportación: informar los cargos y los motivos que los justifican; posibilidad de oponerse a los cargos; ofrecer asesoría legal, intérprete y asistencia consular; opción de recurrir la decisión con efectos suspensivos; ejecución de la medida solo cuando esté en firme la decisión.

- Está prohibida la expulsión hacia territorio donde peligre la vida o la libertad de cualquier extranjero, o hacia un territorio de donde luego pueda trasladarse a otro lugar donde sematerialicen esos riesgos.

- El derecho de los niños, niñas y adolescentes a la protección de la familia y al disfrute de ésta prevalece en los casos en que se contemple la expulsión o deportación de los progenitores, salvo por razones que atiendan al interés superior del niño. Este criterio no es absoluto, y acepta ponderación, siempre y cuando se apliquen los criterios de idoneidad, necesidad yproporcionalidad.

- Procedimientos que deben adoptarse para la concesión del asilo: reconocer el efecto declarativo de la condición; principio de no devolución como piedra angular de la protección; imposibilidadde rechazar a una persona en la frontera.

Sobre la privación de la libertad en procesos migratorios y el alojamiento de niños, niñas y adolescentes en instituciones especificas

- La detención en caso de situación migratoria irregular nunca debe darse con fines punitivos. Unapolítica que no considere alternativas menos restrictivas es considerada arbitraria.

- Las medidas privativas de la libertad en procesos migratorios contra niños, niñas y adolescentes carecen de necesidad. Por ello, cualquier medida de protección que se aplique deberá representarun menor nivel de lesividad que aquel que tiene una medida privativa de la libertad. 
- Las medidas no privativas de la libertad deberán ser consideradas de aplicación prioritaria, mientrasque el alojamiento en instituciones donde se limite la libertad deberá ser excepcional.

- Sobre los centros donde sean alojados los niños, niñas y adolescentes en situación migratoria, precisa la Corte que deberán respetar el principio de separación entre menores de 18 ańos y adultos, salvo en aquellos casos en que el confinamiento sea de la madre y padre junto con sus hijos e hijas. En esos eventos, la separación del núcleo familiar solo puede darse por la necesidadde salvaguardar el interés superior del niño, nińa o adolescente.

- Sobre los centros de alojamiento, señala la Corte que éstos debe tener una modalidad abierta que no signifique la restricción de la libertad, y deben garantizar la protección integral de los niños, niñas y adolescentes, para lo cual deberán garantizar una serie de servicios, tanto básicos como aquellos que permiten el esparcimiento y el desarrollo cultural y social. Igualmente, deberáfacilitarse el acceso a servicios educativos por fuera de la institución.

- Mayor apremio de la asistencia jurídica para niños, niñas y adolescentes que vean restringida su libertad en situaciones migratorias.

\section{REFERENCIAS}

- Beloff, Mary Ana. Los derechos del niño en el sistema interamericano. Buenos Aires, Artes gráficas Candil, 2009.

- Castillo Santiago, Rolando, "La alienación parental: conducta que atenta la salud”. En La Convención sobre los derechos del niño a debate 30 años después, ed. por Antonio Salvador Jiménez Hernández, 534-535. México: CIPI Infancia, 2019.

- Constitución Política de los Estados Unidos Mexicanos, http://www.diputados.gob.mx/LeyesBiblio/pdf/1_201219.pdf

- Convención sobre los Derechos del Niño, https://www.ohchr.org/sp/professionalinterest/pages/crc.aspx

- Corte I.D.H., Caso de la masacre de Mapiripán vs. Colombia... párr. 162.

- Corte I.D.H.: Caso Bulacio vs. Argentina. Sentencia de 18 de septiembre de 2003. Serie $\mathrm{C} \mathrm{N}^{\circ} 100$, par. 134, 55.

- Corte I.D.H.: Caso de las Niñas Yean y Bosico vs. República Dominicana. Excepciones Preliminares, Fondo, Reparaciones y Costas. Sentencia de 8 de septiembre de 2005. Serie C No 130, par. 134, 59; Cfr. Corte I.D.H.: Condición Jurídica y Derechos Humanos 
del Niño. Opinión Consultiva OC-17/02 de 28 de agosto de 2002.

Serie $\mathrm{A} \mathrm{N}^{\circ} 17$, pars. 56, 57 y 60.

- Corte Interamericana de Derechos Humanos y Fondo de las Naciones Unidas para la Infancia, Violencias contra niñas, niños y adolescentes en América Latina y el Caribe, (San José: Corte Interamericana de Derechos Humanos y Fondo de las Naciones Unidas para la Infancia, 2019), 58.

- Corte Interamericana de Derechos Humanos, Cuadernillo de Jurisprudencia de la Corte Interamericana de Derechos Humanos No. 14 : Igualdad y no discriminación, (San José: : Corte IDH, 2019), 11, acceso el 15 de enero de 2020, http://www.corteidh.or.cr/sitios/libros/todos/docs/cuadernillo14.pdf https://doi.org/10.24215/25251678e287

- Fernández de la Reguera Ahedo, Alethia. et al., Caravanas migrantes: las respuestas de México. Serie Opiniones Técnicas sobre Temas de Relevancia Nacional. México: Universidad Nacional Autónoma de México, 2019.

- González Oviedo, Mauricio. Derechos de la niñez y la adolescencia. Costa Rica: Comisión Nacional para el Mejoramiento de la Administración de Justicia, 2001, 35. Acceso 15 de enero de 2020.

https://www.unicef.org/costarica/docs/cr_pub_Antologia_derechos_NNA_Escuela_Judicial.pdf

- Jaggli, Marissa A.. Menores en riesgo social. Córdoba: Mediterránea, 2007.

- Jiménez García, Joel Francisco. El derecho del menor. México: Universidad Nacional Autónoma de México, (2012): 147-148.

- ONU. Comité de los Derechos del Niño, Observación General No. 5 de 27 de noviembre de 2003, párr. 12.

- Olea, Helena. et al. El Sistema Interamericano de Protección de los Derechos Humanos y los derechos de las poblaciones migrantes, las mujeres, los pueblos indigenas y los niños, niñas y adolescentes. San José: Instituto Interamericano de Derechos Humanos, 2004.

- Tesis 1a. CCC/2015, Décima Época, Primera Sala, Semanario Judicial de la Federación, 09 de octubre de 2015, bajo el rubro: BULLYING ESCOLAR. EXISTE UN DEBER DE DEBIDA DILIGENCIA DEL ESTADO PARA PROTEGER Y GARANTIZAR LOS DERECHOS DE LOS MENORES DE ACUERDO CON EL PRINCIPIO DE SU INTERÉS SUPERIOR. 
- Tesis 1a. LXXVI/2013, Décima Época, Primera Sala, Semanario Judicial de la Federación y su Gaceta, Libro XVIII, marzo de 2013, t. 1, p. 887; bajo el rubro: INTERÉS SUPERIOR DE LA NIÑEZ. EL ARTÍCULO 4O. PÁRRAFO OCTAVO, DE LA CONSTITUCIÓN POLÍTICA DE LOS ESTADOS UNIDOS MEXICANOS, REPRESENTA UN PUNTO DE CONVERGENCIA CON LOS DERECHOS DE LA INFANCIA RECONOCIDOS EN TRATADOS INTERNACIONALES.

- Tesis aislada 1a. CXLI/2007, registro de IUS 172003, publicada en el Semanario Judicial de la Federación y su Gaceta, Novena Época, tomo XXVI, julio de 2007, página 265, cuyo rubro es "INTERÉS SUPERIOR DEL NIÑO. SU CONCEPTO".

- Tesis aislada 1a. XLVII/2011, registro de IUS 162354, publicada en el Semanario Judicial de la Federación y su Gaceta, Novena Época, tomo XXXIII, abril de 2011, página 310, "INTERÉS SUPERIOR DEL NIÑO. ES UN PRINCIPIO DE RANGO CONSTITUCIONAL IMPLÍCITO EN LA REGULACIÓN DE LOS DERECHOS DE LOS MENORES PREVISTOS EN EL ARTÍCULO 40. CONSTITUCIONAL”.

- Tesis aislada 1a. XV/2011, registro de IUS 162807, publicada en el Semanario Judicial de la Federación y su Gaceta, Novena Época, XXXIII, febrero de 2011, página 616, cuyo rubro es "INTERÉS SUPERIOR DEL NIÑO. FUNCIÓN EN EL ÁMBITO JURISDICCIONAL".

- Tesis aislada 1a. CXXI/2012 (10a.), registro de IUS 2000989, publicada en el Semanario Judicial de la Federación y su Gaceta, Décima Época, Libro IX, junio de 2012, Tomo 1, página 261, cuyo rubro es "INTERÉS SUPERIOR DEL MENOR. SUS ALCANCES Y FUNCIONES NORMATIVAS”.

- Tesis aislada 1a. CXXII/2012 (10a.), registro de IUS 2000988, publicada en el Semanario Judicial de la Federación y su Gaceta, Décima Época, Libro IX, junio de 2012, Tomo 1, página 260, cuyo rubro es "INTERÉS SUPERIOR DEL MENOR. SU FUNCIÓN NORMATIVA COMO PRINCIPIO JURÍDICO PROTECTOR”. Ver, en un sentido similar, la tesis aislada P. XLV/2008, registro de IUS 169457, publicada en el Semanario Judicial de la Federación y su Gaceta, Novena Época, tomo XXVII, junio de 2008, página 712, cuyo rubro es "MENORES DE DIECIOCHO AÑOS. EL ANÁLISIS DE UNA REGULACIÓN RESPECTO DE ELLOS DEBE HACERSE ATENDIENDO AL INTERÉS SUPERIOR Y A LA PRIORIDAD DE LA INFANCIA". 
- Tesis aislada 1a. CXXIII/2012 (10a.), registro de IUS 2000987, publicada en el Semanario Judicial de la Federación y su Gaceta, Décima Época, Libro IX, junio de 2012, Tomo 1, página 259, cuyo rubro es "INTERÉS SUPERIOR DEL MENOR. SU FUNCIÓN NORMATIVA COMO PAUTA INTERPRETATIVA PARA SOLUCIONAR CONFLICTOS POR INCOMPATIBILIDAD EN EL EJERCICIO CONJUNTO DE LOS DERECHOS DE LOS NIÑOS”.

- Valenzuela Reyes, María Delgadina. Derechos Humanos de los niños y las niñas. México: Porrúa, 2013. 


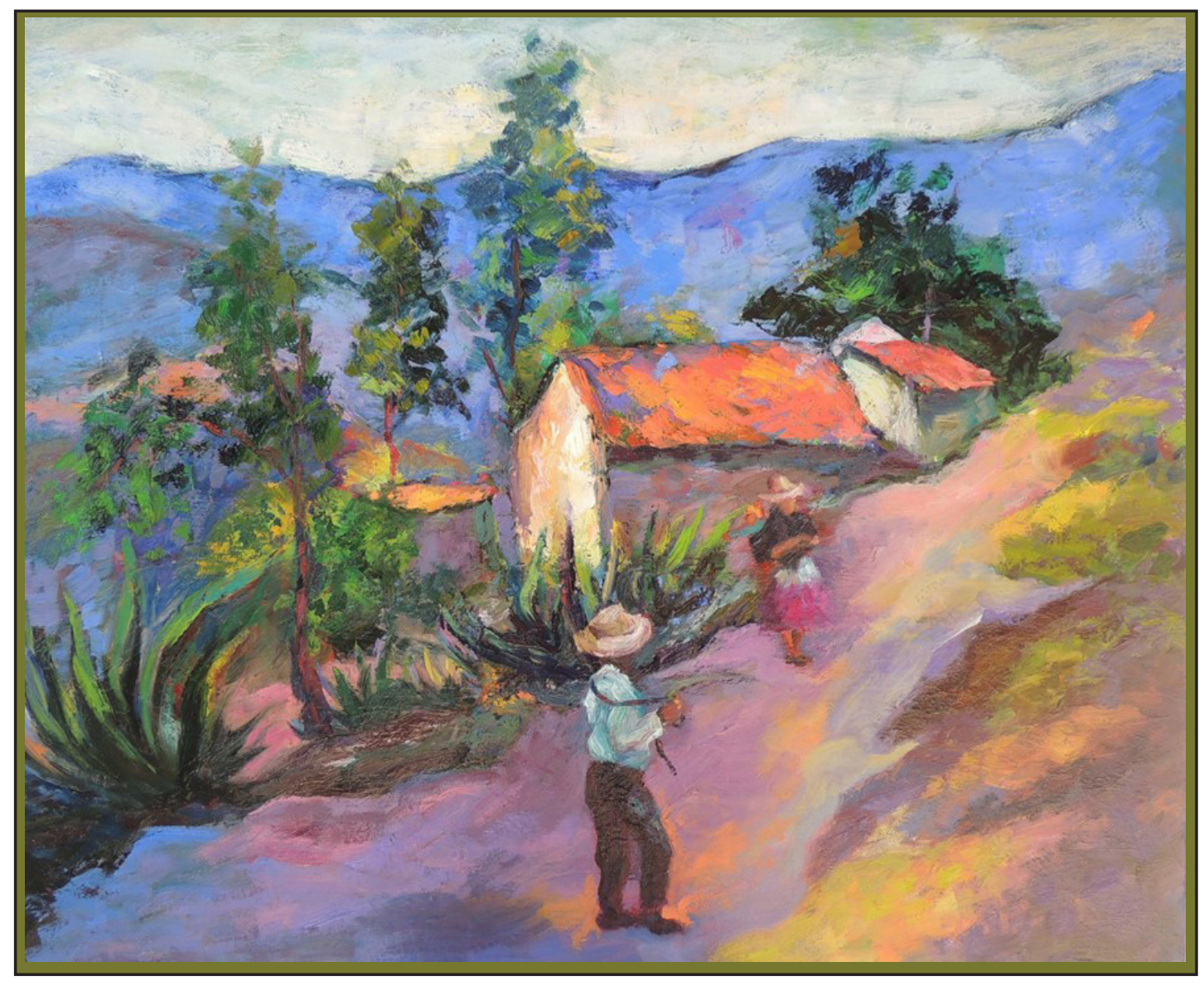

Paisaje de San Cruz. Óleo sobre lienzo 65 x $81 \mathrm{~cm}$. Sonia Estrada Melgarejo (pintora peruana, Ancash) 